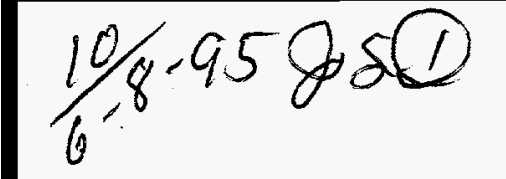

\title{
DIAGNOSTIC BEAM PULSES FOR MONITORING THE SLC LINAC
}

\author{
F.-J. Decker, M. Stanek, H. Smith, F. Tian, \\ Stanford Linear Accelerator Center*, Stanford University, Stanford, CA 94309 USA
}

\begin{abstract}
The Stanford Linear Collider is a pulsed machine with a repetition rate of $120 \mathrm{~Hz}$. By using fast devices such as kickers and triggers, individual pulses can be modified, measured and diagnosed, and then dumped to avoid any background in the experiment. For more than five years, a diagnostic pulse has been used to kick the beams onto offaxis screens at the end of the linac every 6 seconds. This provides a visual monitor of the beam size and loses about $0.14 \%$ of the rate or two minutes a day. The sensitivity of the linac optics to temperature and phase variations makes it desirable to monitor the phase advance between different locations in order to make local corrections. In principle, the feedback systems can measure the phase advance using the natural jitter of the beam. In practice, the phase jitter of the beam with respect to the rf may dominate the betatron jitter and distort the measurement. By using a large induced betatron oscillation, the two effects can be separated. To improve the monitoring of phase advance, a small kicker at the beginning of the linac is fired every few seconds and the orbit of this particular beam pulse measured and analyzed. The sensitivity, the measured variation and the correction scheme will be discussed.
\end{abstract}

\section{INTRODUCTION}

The lattice of the SLC linac can be studied by launching betatron oscillations and comparing the phase advance to the model. Since it takes explicit beam time this task is not done very frequently. This can cause bad setups for example back-phases klystrons, which lead to an oscillating feedback and therefore generate a second generation problem, instead of fixing the original problem. Two solutions are investigated, first to speed up the process, or to get a continuous measurement done by using a small fraction of the beam pulses to monitor the daily behavior. The later one will be discussed by describing the trigger setup, data taking and data analysis, which showed the variations of $\pm 60^{\circ}$ in betatron phase advance.

*Work supported by the Department of Energy, contract DE-AC03-76SF00515.

\section{TRIGGER SETUP}

During the last run every hour a kicker was activated in the RTL (ring-to-linac) section. It fired every 6 seconds and this pulse was readout by the linac BPMs. This was exactly one of the pulses which are anyway kick onto the off-axis screens in sector 30 at the end of the linac. The screen spots were disturbed since the beam was half lost on the nearby collimators. The average of 10 kicked and 10 non-kicked orbits was saved as a reference orbits. Since the software routine was put on top of an existing one, the wire macro loop, it wasn't always running. Fig. 1 shows a couple of typical difference orbits over a day.

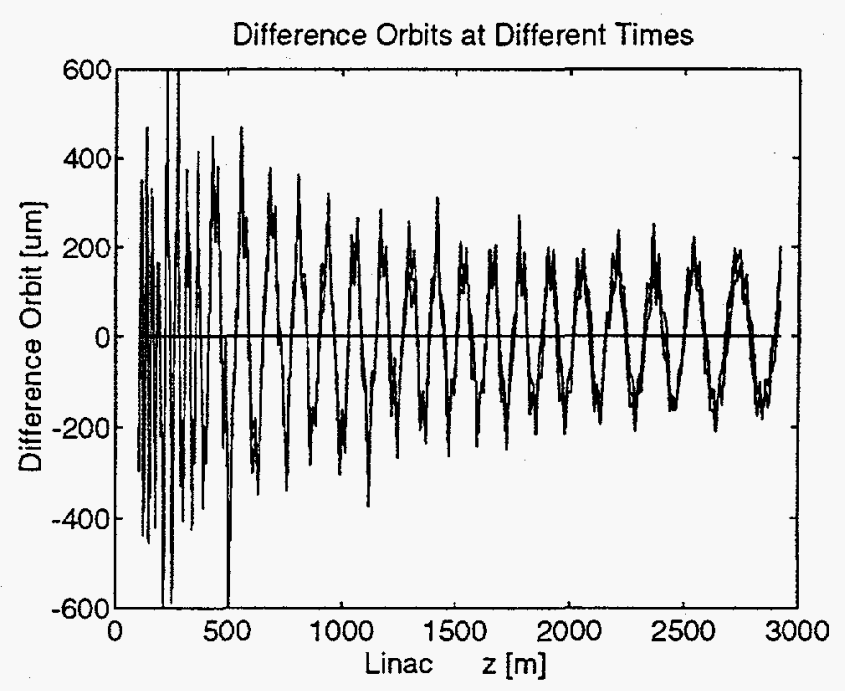

Fig. 1: Betatron oscillations at different times. The different orbits show phase advance changes over the course of a day. At the end the beam is partly lost at collimators and then kicked onto off-axis screens.

\section{DATA READOUT}

These reference orbits were read out with a small MATLAB program which generated all the differences orbits. By fitting small sections of this oscillation (15 BPMs) to a betatron oscillation, $y$ and $y^{\prime}$ were generated locally.

Presented at the 16th IEEE Particle Accelerator Conference (PAC 95) and International Conference on 
The phase advance was calculated with the following formula:

$$
\dot{\phi}=\arctan 2\left(\beta y^{\prime}+\alpha y, y\right)
$$

Plotting the phase advance versus the length $z$ of the linac, can give some hints of the setup of the lattice and local problems. In Fig. 2 this plot is done for a period when a change in the linac setup from SLC to FFTB occurred. The changes were: Change from a split tune lattice back to SLC-design, less BNS phase offsets, and lower current, plus some not anticipated changes. There is a kink in the top curve (FFTB) near $200 \mathrm{~m}$, which was later found to be the klystron 3-1 with $120^{\circ}$ phase offset. The other kink in the middle of Sector 2 in the split tune lattice data is not yet understond.

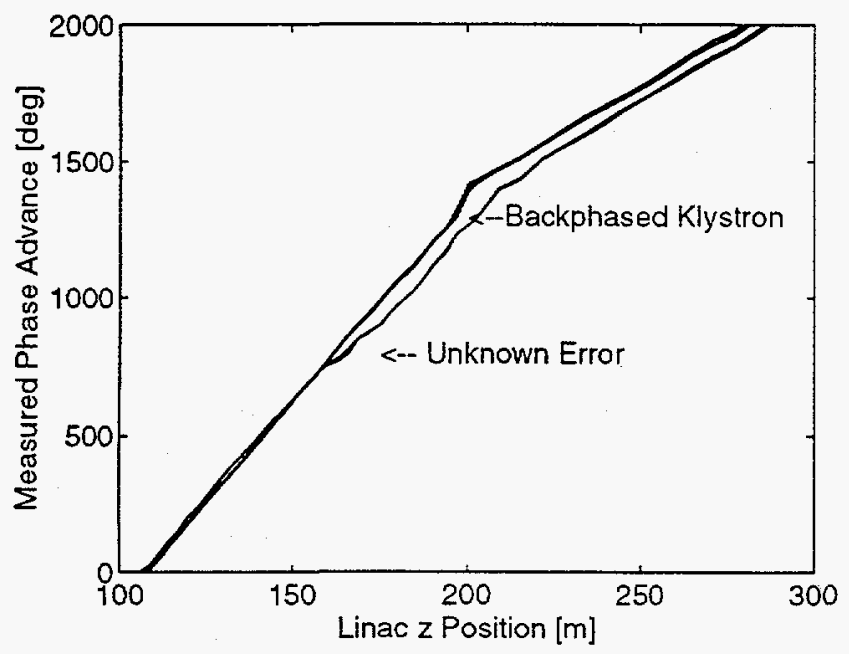

Fig . 2: Betatron phase advance.

The phase advance in the beginning of the linac shows a clear change for data before and after the switch to FFTB. The expected gradual change is overwhelmed by two distinct problems.

The mean phase advance of many days was subtracted and the different phase advance differences for one set up are plotted versus the linac location (Fig. 3). The biggest changes occur in the beginning of the linac. Near $400 \mathrm{~m}$ is a place with much less variation, which means that one error is canceling another one. The fast oscillations are coming from a mismatch compared to the average phase (see below).

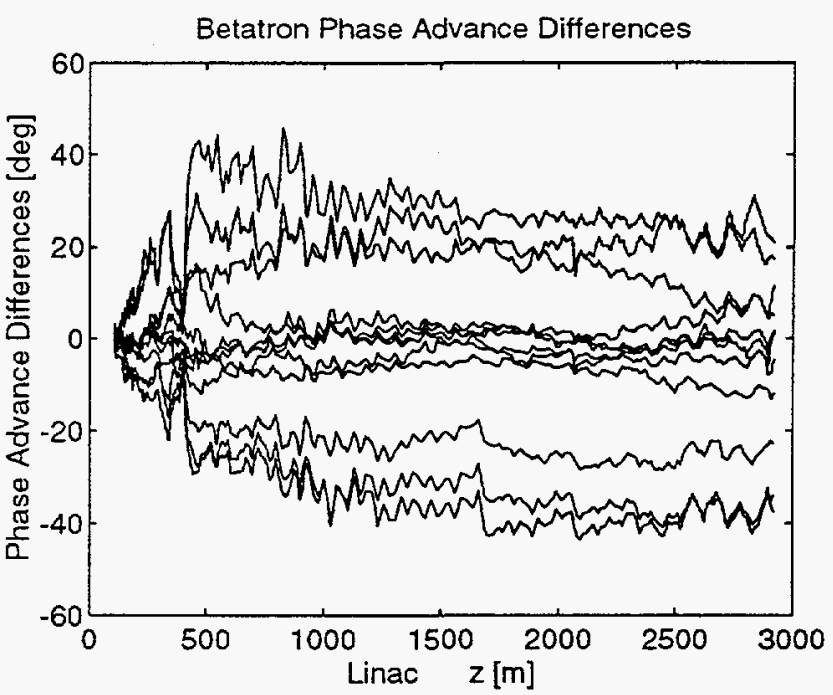

Fig. 3: Phase advance differences for different times. The phase advance changes mainly in the beginning of the linac up to the first $1000 \mathrm{~m}$. while it stays quite stable afterwards.

\section{DATA ANALYSIS}

\section{A. Tenperature variations}

Besides looking carefully at the data to localize specific problem region, the data were analyzed for daynight variations. Therefore each BPM was plotted versus time and compared with the outside temperature. The correlation with the temperature can be achieved by calculating the temperature coefficient which is equivalent with the slope of data versus temperature. This method has the advantage that the change can be compared at different day with different temperature swings.

Initial trends of a big correlation in the beginning of the linac, couldn't be confirmed, due to less temperature variation which gave only a non-significant correlation. Later at the end of the FFTB run when the temperature changes were high there was also no correlation. But that time it could have been fixed by a new SLED tuning procedure [1].

\section{B. Comparison to SLC-design lattice}

A fixed phase advance from the model for the SLCdesign lattice was taken and compared with the measured phase advance to for different time periods. The following observations are initially expected: 


\section{DISCLAIMER}

This report was prepared as an account of work sponsored by an agency of the United States Government. Neither the United States Government nor any agency thereof, nor any of their employees, makes any wartanty, express or implied, or assumes any legal liability or responsibility for the accuracy, completeness, or usefulness of any information, apparatus, product, or process disclosed, or represents that its use would not infringe privately owned rights. Reference herein to any specific commercial product, process, or service by trade name, trademark, manufacturer, or otherwise does not necessarily constitute or imply its endorsement, recommendation, or favoring by the United States Government or any agency thereof. The views and opinions of authors expressed herein do not necessarily state or reflect those of the United States Government or any agency thereof. 


\section{DISCLAIMER}

Portions of this document may be illegible in electronic image products. Images are produced from the best available original document. 
1. High current beam loading

a) more phase for strong BNS "damping"

b) flat for auto-phasing

c) less phase for weak BNS

2. Split tune lattice: less phase for $\mathrm{e}^{-}$

3. good agreement for low current (FFTB).

Figure 4 shows the phase advance difference to the SLC design. The deviations from the expected behavior could come from localized errors. Another fact is that the BNS phase is given to the energy management package, which then lowers the magnet strength a for stronger BNS. This has the effect that the head of the bunch is higher in energy and therefore has a slower phase advance.

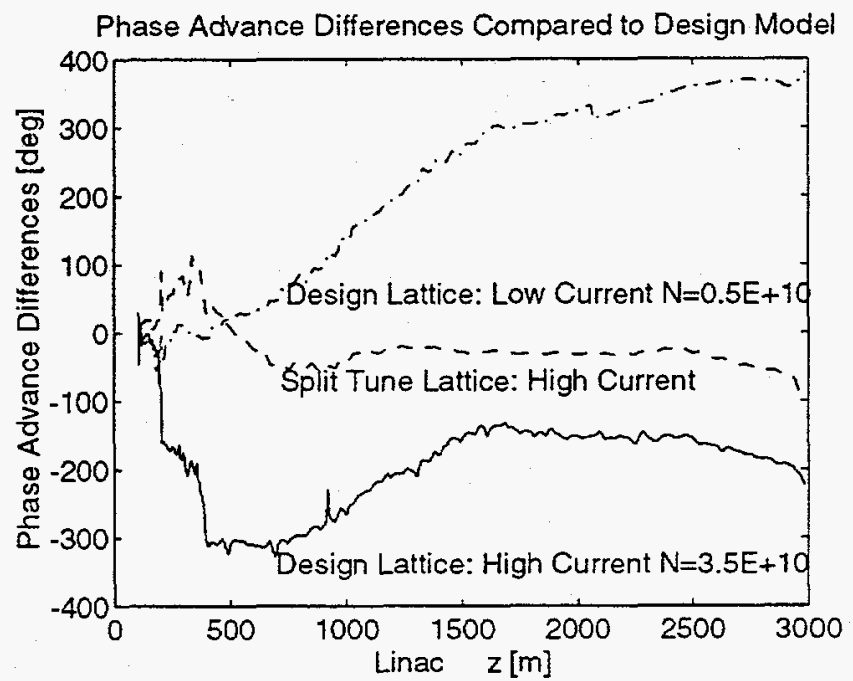

Fig. 4: Three phase advance differences to SLC design. The measurement shows: About $360^{\circ}$ change over the length of the linac for FFTB, two big changes in Sector transition $200 \mathrm{~m}$ and $400 \mathrm{~m}$ where the quadrupole spacing changes by a factor of two for high current design lattice case, and some positive phase for the split tune case.

\section{Measurement resolution}

The phase differences for ten orbits in a short time span will give the resolution in phase for this method. It is about $5^{\circ} \mathrm{rms}$ in phase advance over half an hour. Taking the difference of only two phase advances shows some oscillatory behavior in the phase advance, which is an indication of the a mismatch, with different, faster and slower, phase progress than a match case (see Fig. 5).

\section{CORRECTION SCHEMES}

The first order correction which is envisioned is over the operators. Therefore the off-line code has to get on-line,

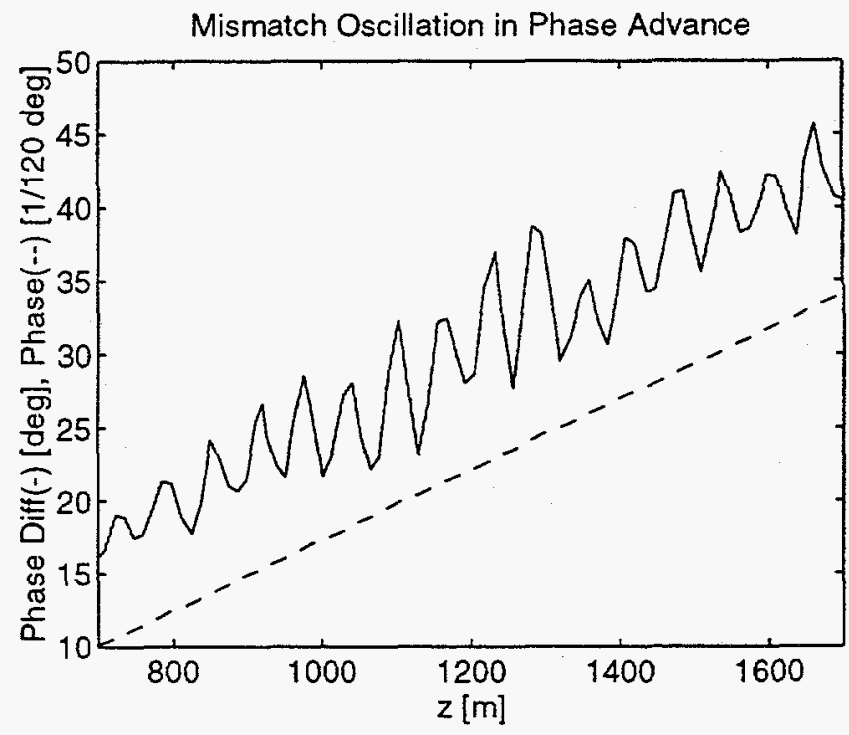

Fig. 5: Phase and phase advance difference.

In the second quarter of the linac the phase advance difference (solid) of two beam pulses shows a $\pm 5^{\circ}$ oscillation. The 16 oscillations occur during 8 $\left(=24^{\circ} * 120 / 360^{\circ}\right)$ betatron wave length (dashed) and indicate a mismatch.

that a single phase advance can be compared to a reference phase advance, saved or design (like the reference orbits now). The next step would be to "steer" (adjust) the quadrupoles to that reference, which would be local, comparable to the existing global energy management (LEM). Further steps might be the use of the phase information for steering feedbacks or even the automation of the above procedure via a local LEM feedback.

\section{CONCLUSION}

The phase advance difference, measured with diagnostic pulse, is a precise tool to detect any changes or deviations of the order of $\pm 5^{\circ}$ in betatron phase. The noninvasive character of the diagnostic pulse makes it possible to get that information during the normal colliding beam run. The off-line data processing has to get on-line for the next run.

\section{REFERENCES}

[1] F.-J. Decker et al., Effects of Temperature Variation on the SLC Linac RF System, PAC95, Dallas, May 1995. 